\title{
A REVIEW ON AQUA WATER DELIVERY SYSTEM
}

\author{
Archana K Arjunan, Binitha Sabu, Josna Johnson, \\ UG Scholars, Dept. of CSE, MBITS \\ Midhun Mathew, \\ Head of the Department, CSE, MBITS
}

\begin{abstract}
Water Distribution and Management is a web application which enhances and emphasizes the way water is distributed and maintains the quality. The people who are in search of water for their regular use can use this application. Thus, provide water to different areas with packages at affordable rates. The users can order water. The customers can make payments online. The system proposes an android application through which accurate delivery of water can be done through tracking water suppliers. It will reduce the transportation delay and delivery reaches customers safely. The users can provide feedback based on the quality of water. Sentiment analysis is used to check the polarity of sentences to know whether the review is positive or negative. It helps to analyze the human reactions and opinions and thus predicting the quality of the water plant.
\end{abstract}

Index Terms - Sentiment analysis, water can delivery, GPS, online payment

\section{INTRODUCTION}

We propose a system for managing the drinking water system that overcomes shortage of water due to limited water resources and high demand. It is a network of plants. The water can be taken from any of the plants. The customers can regularly order the water on daily, monthly or weekly basis. There will be an offer for the customers for regular booking. The people can pay the cash for water online. It also provides lots of advantages like customize orders, enhanced user interface payment orders etc. The registered drivers can accept delivery orders and make accurate delivery of water by tracking. It will provide job opportunities to the employees. After the water is utilized by the users, they can give feedback too, as feedback is also an interface part of the system as the user satisfaction and any kind of complaints can be informed. According to the feedback of the customers, quality of the water will be analyzed using sentiment analysis in data mining. Sentiment analysis is included in this system for analyzing statements and obtains information about them. Data is collected from user's feedback. By using a Vehicle Tracking System the raw data can be extracted through devices mounted on vehicles, analyzed and formatted for making further conclusions related to tracking. Sentiment analysis is also known as opinion mining. Not only in case of business, have we often seen people give opinions as review of movies which is also very important for a film maker to know audiences' responses. Now-a-days the social media such as blogs or various social platforms has increased the interest for sentiment analysis. Without people's opinion we cannot determine whether there is any need for change. Sentiment analysis is required in this space. Text based sentence analysis process is to know the polarity of sentences ie, whether the reviews given by people are positive or negative.

\section{RELATED WORK}

Availability of good quality water is the most essential need in our life. Nowadays the water distribution is done manually. The existing system is old fashioned and there is no usage of commonly used technology like the internet, android and digital money. The working procedure in those cities is where a customer calls or drives down to the shops to order the water containers by providing address and details manually. Then the delivery person delivers the order to the customer's door step. The customers are paying the money by giving it to the route boys or directly to the agent. This system is completely offline. There will be repeated calls from or to customers, if multiple orders are placed from the same locality. So, the delivery person needs to travel multiple times to the same locality. There is no track of orders and e-transactions. We are unable to know whether the delivery boy delivers the order.

\section{BACKGROUND}

\section{A. Accounts and Management}

Accounts and Management mainly focus on the registration process and verification. The Water plant, agency and user can register into the system through the registration page. They can register into the system by providing essential details. The agency is verified by the 
government. The Water plant will be verified by the agency by using license number and document. The privacy settings were related to the individual information. The information about agency, water plant and user can thus be viewed and edited.

\section{B. Water Distribution and Management}

The main purpose of water distribution and management is to deliver water to the customers with appropriate quality. The agency will send an application towards the agency. The customers search for an agency that provides service on the distribution of water. Customers will get quality water from good water plants through agency. Packages will be set by the agency at discounted rates. Packages can be provided on a weekly, monthly or yearly basis. The agency also provides individual packages for the customers who want water. Depending on the packages set by the agency, the customers will order the water. The order will be verified by the agency.

\section{Employee Management}

The agency will assign employees for the distribution of water. The agency will manage the order allocation and allocate employees for the delivery. The employees will view the delivery location using google map and track the delivery which helps to minimize the driving time and fuel consumption by finding the most efficient route. It will also reduce the transportation delay. Thus the delivery reaches to customers safely. It provides job opportunities to the drivers.

\section{Stock Module}

Stock allows the water to be tracked across the entire supply chain. The stock management will manage from the order placed with the vendor to deliver the water for the customers by mapping the complete journey of the product. The agency will request for the water plant when the stock has been ended.

\section{E. Quality Analyzing}

Sentiment analysis is done using an algorithm that uses text analysis and NLP to classify words as either positive or negative. The customers will provide feedback for the quality of water. Quality analyzing module allows the customer to identify better quality water plants by reading reviews. The feedback is analyzed using sentiment analysis.

\section{PROPOSED SYSTEM}

This is an online system for the distribution of water. The customers, who want water, can use this system effectively. The aim of our system is to regulate and optimize water supply in the areas in which they need them the most and ensure that whenever the request is made. The customer can directly register to the system and can order the water they want. The customer can make payments through the website. A customer who wishes to pay the money can make payments whenever he wants. They are provided with the route maps to locate the customer. It is very easy for a new route boy to distribute. Admin is the person who registers the route boy. The payment is done between the admin and the customer. When the customer's registration is accepted by the admin, he can login to the system by using user ID and password. Users can give feedback about the quality of water from the water plant and service from the agency. By the reviews and feedback, the water plant and agency are rated and they are considered as the good water plant and agency. For the convenience of the delivery person, an API of Google Maps for finding the optimized route is possible. The person can access the system anywhere in one country.

\section{SYSTEM ARCHITECTURE}

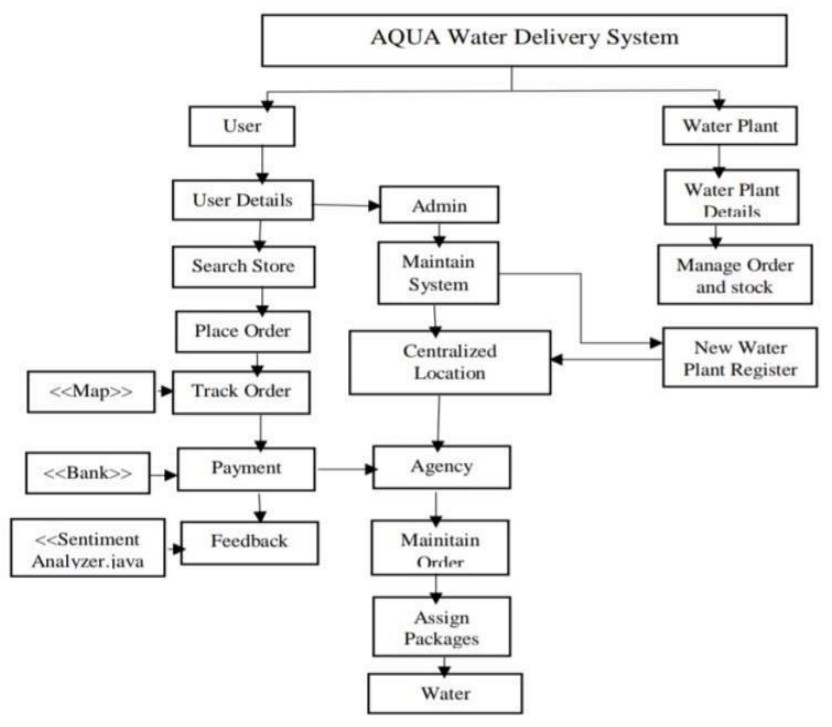

Fig 1: System Architecture Aqua Water Delivery System

\section{ALGORITHM}

1. Accept the feedback from database.

2. Tokenize the sentence into words.

3. Remove stop words.

4. Start a loop for each word in the array.

4.1. Extracted words are checked against positive and negative words in the library

SentimentClassification.java

4.2. If the words matched with stored words.

4.3. If matched with stored negative 
words, negative_count $=$ negative_count+1

4.4. If matched with stored positive words, positive_count $=$ positive_count+1

4.5. Sentiment emotion is checked by score of the words

4.5.1 Total score of positive and negative words calculated

4.5.2 If total score of positive emotion > negative emotion, then type of feedback is positive.

4.5.3 If total score of negative emotion > positive emotion, then type of feedback is negative.

\section{A. Detecting Good or Bad Reviews}

Sentiment analysis is a part of Natural Language Processing (NLP) techniques. It is the process of using NLP, text analysis, and statistics to analyze customer sentiment. This was used on social media posts and customer reviews in order to automatically understand whether users are positive or negative. The goal of this project is to show how sentiment analysis can be performed using NLP.

\section{B. Support Vector Machine}

Support Vector Machines can be very much effective in sentiment clarification. The key is not the algorithm but the pre-processing required. This paper introduces an approach towards classifying texts as positive or negative using SVMs, a well-known and powerful tool for classification of vectors of real-valued features.

\section{Sentiment Analysis}

Sentiment analysis algorithm is for predicting the quality of water plants. Sentiment analysis is also known as open mining. If the spelling check of the sentence is right, CFG checking is done. Otherwise the spelling of the sentence is wrong. If CFG is correct, sentence emotion is done. If the emotion is positive the positive count increases otherwise negative count increases. If CFG checking is incorrect, the wrong sentence format is detected. An error message is generated as the program gets input as a NULL sentence. If the count of positive emotion is greater than negative emotion, then the result is positive. Otherwise the result is negative.

\section{CONCLUSION}

The Aqua Water Delivery System helps the water to be delivered to the customer at any places effectively.
Customers can guarantee better quality water which prevents diseases spreading through contaminated water. Regular, monthly or weekly packages will be set at discounted rates. The focus of this project is to make accurate delivery of water by tracking water suppliers. Customers can identify water quality by reading reviews. Thus the quality of water is assured by feedback of customers through sentiment analysis.

\section{REFERENCE}

[1] Congcong Sun, Gabriela Cembrano, VicencPuig, (2018), “Cyber-Physical Systems for Real-time Management in the Urban Water Cycle", International workshop on Cyber-Physical Systems for Smart Water Networks, 10.1109/CySWater.2018.00008.

[2] Praveen Kumar, N. H. RenitaSwezalGonsalves, (2017) "Online Android Application For Ordering Water And Delivery Management System”, (Pg 98-99).

[3] Biswarup Nandi, MousumiGhanti, Souvik Paul, (2017) "Text Based Sentiment Analysis", Proceedings of the International Conference on Inventive and Informatic, 10.1109/ICICI.2017.8365326

[4] Dangling Ma, Jian Cui, (2017), "Design and Realization of Water Quality Information Management System Based on GIS", 10.1109/ISWREP.2011.5893122.

[5] SandisDejus, Rasa Bruzgiene, AnatolijsZabasta, NadezdaKunicina, (2018), "Quality-Focused Data Delivery in Wireless Sensor Network for Drinking Water Distribution System, 10.1109/AIEEE.2018.8592151.

[6] NamanSatiya, VinitVaru, AakankshaGadagkar, DarshanSaha, (2017), “Optimization of Water Consumption using Dynamic Quota based Smart Water ManagemetSystem”,10.1109/TENCONSpring.2017.807007 5.

[7] M Suresh, U. Muthukumar, Jacob Chandapillai, (2017),"A Novel Smart Water-Meter Based on IoT and Smartphone App for City Distribution Mnagement", 10.1109/TENCONSpring.2017.8070088.

[8] SujithDeshpande, TejasBerhate, KapilMundada, (2015), "Design and Development of Cost Effective and Intergrated Water Distribution System for Residential Establishment", 10.1109/INFOP.2015.7489464.

[9] Rajeev Kumar, Hareesh Kumar, (2015), "Availability and Handling of Data through GPS Device: In Tracking a Vechicle”, 10.1109/IAdCC.2014.6779328. 
[10] Yi Zhou, Tiesong Hu, (2009), "Flexible Design Of Delivery Capacity in Urban Water Distribution System" 10.1109/ICMSS.2009.5304756,

[11] SeokJu Lee, GirmaTewolde, Jaerock Kwon, (2014), "Design and Implementation of Vehicle Tracking System using GPS/GSM/GPRS technology and Smartphone Application", 10.1109/WFIoT.2014.6803187.
[12] SanthoshGautamKashid, Sanjay A Pardeshi, (2014), "A Survey of Water Distribution System and new approach to Intelligent Water Distribution System", 10.1109/CNSC.2014.6906645. 